\title{
Multimodality Medical Image Fusion using Rotated Wavelet Transform
}

\author{
S. Chavan ${ }^{1}$, A. Pawar ${ }^{2}$ and S. Talbar ${ }^{3}$ \\ ${ }^{1}$ Don Bosco Institute of Technology, Kurla(W), Mumbai, Maharashtra, India 400070 \\ ${ }^{2}$ Smt. Kashibai Navale Medical College and General Hospital, Narhe, Pune, Maharashtra, India 411041 \\ ${ }^{3}$ SGGS Institute of Engineering and Technology, Nanded, Maharashtra, India 431606 \\ \{1 satyachavan@yahoo.co.in; ${ }^{2}$ abhijeetpawar.rad@gmail.com; ${ }^{3}$ sntalbar@yahoo.com\}
}

\begin{abstract}
This paper presents a novel approach to multimodality medical image fusion for better visualization of lesions and calcification. The algorithm utilizes source modalities as Computed Tomography (CT) and Magnetic Resonance Imaging (MRI). It is a feature based fusion technique in which Rotated Wavelet Transform (RWT) is used for extraction of edge-related features from both the source modalities. These features are used to create new frequency domain plane using maxima and entropy based fusion rules. The fusion process is useful in the analysis of the lesions for diagnosis, treatment, and post treatment reviews. The proposed technique is evaluated on the pilot study sets using objective analysis parameters like entropy, root means square error, edge quality measure, mean structural similarity index measure, etc. The fusion results of the proposed technique are compared with the existing fusion algorithms. The subjective analysis of the fused images by radiologists reveals that the fused images using RWT technique are superior and present all relevant anatomical structures.
\end{abstract}

Keywords: Medical Image Fusion, Rotated Wavelet Transform, Discrete Wavelet Transform, Cancer Treatment, Radiotherapy, RWT filter mask, Fusion Parameters.

\section{Introduction}

Medical image fusion plays a significant role in the cancer patient management system. Its role spreads from the diagnosis, treatment to review of patient's response. In cancer patient's cases, radiologists prefer different modalities for diagnosis as one modality may not provide sufficient information about the disease and its complete spread. Computed tomography (CT) and plain radiographs (X-rays) are used to visualize bony structures and hard tissues. Magnetic resonance imaging (MRI) and functional magnetic resonance imaging (fMRI) are preferred for soft tissue visualization and activity maps, respectively. Positron emission tomography (PET) and single-photon emission computed tomography (SPECT) are the modalities for diagnosis and treatment using nuclear medicine [7]. All these modalities are acquiring information from human body non-invasively and have different physics of acquisition process. Each modality captures only few information about the disease and may not provide a complete picture of the disease. There have been attempts to capture information using two modalities with a single machine. PET-CT is now common in nuclear medicine treatment of cancer patients. PETMRI [24] and CT-MRI [17] machines are proposed, but there are many hardware issues and it is still a dream.

In radiotherapy treatment, $\mathrm{CT}$ is used as a major modality due to treatment plan demands electron density representation [20]. MRI is used for better visualization of soft tissues and tumor contrast. The contouring of lesions and organs are necessary information presented by MRI, whereas actual treatment is based on CT scans. There are a lot of efforts in this process. Obviously, there is a need to have both the CT and MRI content in one frame useful for contouring and treatment planning. Such software solution which presents both the information in a single frame is called multimodality medical image fusion (MMIF). It uses relevant and complementary content from CT and MRI to generate new plane without visual distortion. MMIF is applicable in almost all modern health care practices. It includes diagnosis, treatment plans, follow-up reviews, etc.

Multimodality medical image fusion is very active and developed area of research. Many researchers have developed algorithms for fusion in the last decade. The extensive survey of MMIF is presented by James et al. [6]. It explains the fusion methods based on modalities used, methodologies, and infected organ based techniques. MMIF is widely classified in three main categories viz. pixel based fusion, feature based fusion and decisionbased fusion [22]. Pixel based fusion approach is easy to implement and can be applied in both spatial as well as

B. Iyer, S. Nalbalwar and R. Pawade (Eds.)

ICCASP/ICMMD-2016. Advances in Intelligent Systems Research.

Vol. 137, Pp. 627-635.

(C) 2017- The authors. Published by Atlantis Press

This is an open access article under the CC BY-NC license (http://creativecommons.org/licens)es/by-nc/4). 
spectral domain. Principal component analysis (PCA), intensity-hue-saturation (IHS), and the Brovey transform techniques have a major limitation in terms of spectral information [12].

The decision-based fusion requires a priori information of modalities and features to be combined. Neural network, fuzzy or genetic based fusion approaches are considered under this type of fusion class.

Feature based fusion involves feature extraction using transforms. It uses extraction of spatial or spectral features to integrate them into the new fused image. Various transforms are used to extract spectral features and combined to create a new spectral frame using appropriate fusion rules. The discrete wavelet transform (DWT) is most popular multiresolution fusion approach. DWT provides much better fusion results as compared to the pyramid transforms and lifting schemes due to its better representation of spatial and spectral information simultaneously [11], [15], [23]. Due to the limitations of DWT, like phase variance, less directionality, and limited spectral representation, complex wavelet transforms performs better for fusion of medical images [2], [16].

There are successful attempts to fuse multimodality medical images using feature extraction with transform followed by training to combine these features. Kavitha et al. presented a neuro-fuzzy approach on features extracted using Integer Wavelet Transform (IWT) [8]. The nonsubsampled contourlet transform (NSCT) with pulse coupled neural network gives good fusion results [3], [4]. The fusion using redundancy discrete wavelet transform (RDWT) and NSCT is presented by Rajkumar et al. [13].

This paper presents a new medical image fusion algorithm using rotated wavelet transform (RWT). The spectral features in different orientations other than DWT are extracted with RWT. The algorithm is tested using CT and MRI images. The fused images are evaluated using subjective and objective analysis.

The paper is presented as follows: Section 2 describes the proposed system with principles of discrete wavelet transform (DWT) and design of rotated wavelet transform (RWT) for fusion. Section 3 gives a comparative analysis of the proposed algorithm with other fusion schemes. Section 4 presents conclusion with the future scope of the work.

\section{Proposed System}

The proposed fusion algorithm is presented as a block schematic in Figure 1. It consists of CT and MRI as source images. These images are first registered using geometric transformations like resizing, rotation, and shifting. The registration of two different modality images is complex and crucial. The performance of fusion process is completely depend on how effectively two images are voxel matched. The slices from CT scan sequence and MRI study sets are selected using anatomical and structural similarities with the help of radiologists. The interactive registration approach is used for registration process in this paper [18].

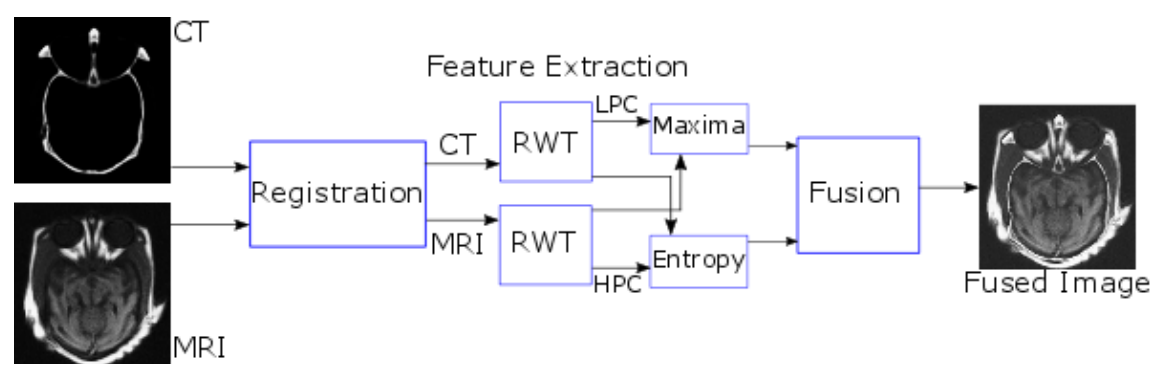

Fig. 1. Block schematic of the proposed system.

These registered source images are decomposed using RWT to extract edge-based features prominently. It is then followed by combining these extracted features using fusion rules namely maxima for low-frequency coefficients and entropy based fusion rule for high-frequency coefficients. New feature plane is created in the frequency domain. The fused image is reconstructed in the spatial domain by applying inverse RWT on this new feature plane. The fused images are evaluated using subjective and objective fusion parameters presented in Section 3 .

\subsection{Discrete Wavelet Transform}

The discrete wavelet transform (DWT) is signal analysis technique presented by Mallat [10]. It represents information using sparse vectors and separates the multiresolution components for further processing. It is defined 
using two basis functions called scaling function $\phi(x)$ and wavelet function $\psi(x)$. DWT decomposition provides both spatial and spectral information simultaneously with better representation of approximate and detailed content from the signal. One dimensional decomposition of signal $f(x)$ using DWT for three levels is shown in Figure 2. The wavelet filter coefficients $h_{1}[n]$ and $g_{1}[n]$ are used as low pass and high pass filter coefficients, respectively. The low pass filtered coefficients are further decomposed in the next level of decomposition. After every level of decomposition, down sampling operation is applied to reduce the redundancy [5].

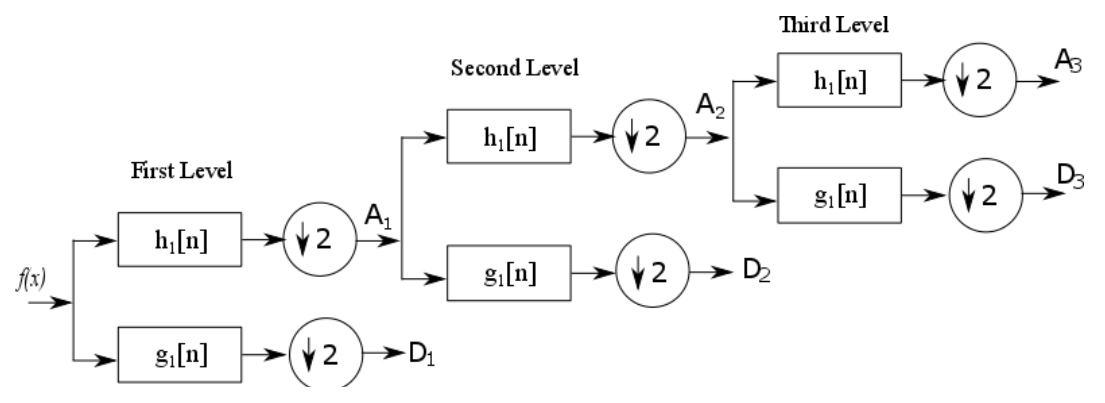

Fig. 2. Three level analysis of discrete time signal using DWT.

The wavelet function $\psi_{a, b}(x)$ is defined as Equation 1. Here, $a, b \in R$ and $a \neq 0$ are constants. The scaling function $\phi_{k}^{j}(x)$ and wavelet basis function $\psi_{k}^{j}(x)$ of DWT are given in Equation 2 and 3, respectively. Here, $j, k \in$ $Z$ and $\phi(x) \in L^{2}(R)$.

$$
\begin{aligned}
& \psi_{a, b}=\frac{1}{\sqrt{|a|}} \psi\left(\frac{x-a}{b}\right) \\
& \phi_{k}^{j}(x)=2^{\frac{j}{2}} \phi\left(2^{j} x-k\right) \\
& \psi_{k}^{j}(x)=2^{\frac{j}{2}} \psi\left(2^{j} x-k\right)
\end{aligned}
$$

DWT helps in presenting signal in frequency domain useful for signal energy spread analysis [5]. The 2-D DWT is the multiresolution representation of an image. It is separable transform in which 1-D DWT is used to achieve 2-D DWT transform. The multiresolution analysis (MRA) presents 2-D data in four sub-bands viz. approximate (AA), horizontal (AD), vertical (DA), and diagonal (DD). This MRA process is repeated on the approximate plane (AA) to get next level of decomposition. In every level, the approximate plane is further processed to get next level [14]. The edge information provided by $2-\mathrm{D}$ DWT is horizontal $\left(0^{\circ}\right)$, vertical $\left(90^{\circ}\right)$, and diagonal $\left(+45^{0}\right.$ and $\left.-45^{0}\right)$. Figure 3 provides a spectral representation of edge information in 2-D DWT decomposition.

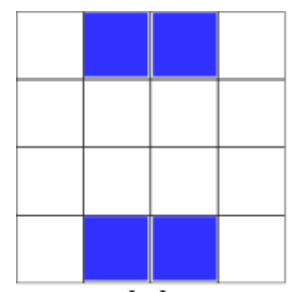

(a)

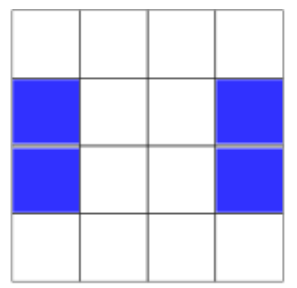

(b)

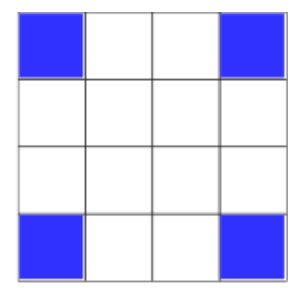

(c)

Fig. 3. The spectrum of 2-D DWT filter masks depicting edge information in sub-bands (a) Horizontal mask $\mathrm{H}_{\mathrm{AD}}\left(0^{0}\right)$, (b) Vertical mask $\mathrm{H}_{\mathrm{DA}}\left(90^{\circ}\right)$, (c) Diagonal mask $\mathrm{H}_{\mathrm{DD}}\left( \pm 45^{\circ}\right)$.

\subsection{Rotated Wavelet Transform (RWT)}

Rotated Wavelet Transform (RWT) is modified version of standard 2-D DWT. The demerits of DWT such as combined diagonal edge information is overcome using this modified RWT. The edge information presented by 2-D DWT is in $\left(0^{0}, 90^{\circ}, \pm 45^{\circ}\right)$ directions. Out of these orientations, $\pm 45^{\circ}$ can be split and presented in two separate orientations with the decomposition using RWT. 
2-D DWT decomposition of an image $f(x, y)$ involves filtering of rows and columns separately by 1-D DWT filter coefficients. Here, $h_{1}[n]$ and $g_{I}[n]$ are commonly used as low pass and high pass filter coefficients, respectively. However, non-separable 2-D DWT filter masks can be used for the same purpose. The process of design of 2-D DWT filter masks is based on products of 1-D scaling and wavelet functions as defined using Equations 4 - 7. These functions are used to generate 2-D filter masks $H_{A A}, H_{A D}, H_{D A}$, and $H_{D D}$ [9]. These 2-D filter masks $\left(\mathrm{H}_{\mathrm{k}}\right)$ are rotated by angle $45^{\circ}$ to design new filter masks called RWT filter masks $\left(H_{k}^{R}\right)$. Here, ' $\mathrm{k}$ ' stands for $A A$, $A D, D A$, and $D D$ which corresponds to four sub-bands. The total size of these filter masks is (2M-1) $\mathrm{x}(2 \mathrm{M}-1)$ for $\mathrm{M}$ number of coefficients in 1-D DWT filter.

$$
\begin{aligned}
& \phi_{A A}(i, j)=\phi(i) \phi(j) \\
& \psi_{A D}(i, j)=\phi(i) \psi(j) \\
& \psi_{D A}(i, j)=\psi(i) \phi(j) \\
& \psi_{D D}(i, j)=\psi(i) \psi(j)
\end{aligned}
$$

Thus, RWT filter coefficients are designed with the help of standard 2-D DWT filter coefficients. Applying these masks on the image, the features oriented in $+45^{\circ}$ and $-45^{\circ}$ are extracted distinctly oriented in $0^{\circ}$ and $90^{\circ}$, respectively. The frequency domain representation of these 2-D RWT filter masks is shown in Figure 4.

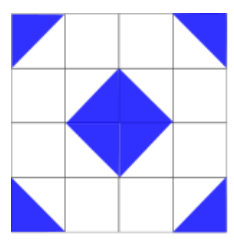

(a)

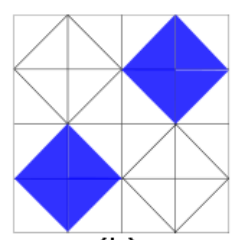

(b)

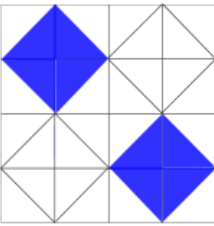

(c)

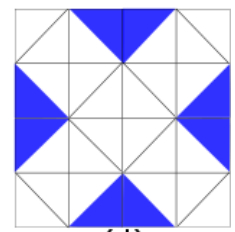

(d)

Fig. 4. Spectrum of 2-D RWT filter mask for edge orientations in sub-bands

(a) Approximate $H_{A A}^{R}$ (No edge) (b) $H_{\mathrm{AD}}^{\mathrm{R}}\left(-45^{0}\right)\left(\right.$ c) $H_{\mathrm{DA}}^{\mathrm{R}}\left(+45^{0}\right)$ d) $H_{\mathrm{DD}}^{\mathrm{R}}\left(0^{0}\right.$ and $\left.90^{\circ}\right)$

\subsection{Fusion Rules}

The paper presents two significant fusion rules used to fuse spectral information. The rules are designed considering the information extraction process. As 2-D RWT provides the feature information in varied orientations as compared with 2-D DWT. Both DWT and RWT provides approximate plane which is a representation of lowfrequency content. Therefore, dominant coefficient out of both the modalities is used for the composite plane. This is achieved using maxima fusion rule defined using Equation 8.

$$
\operatorname{NewPlane} e^{A A}(u, v)=\max \left\{C T^{A A}(u, v), M R I^{A A}(u, v)\right\}
$$

Second fusion rule is for the detailed coefficients selected based on a dominant edge in one of the modalities. The rule is local entropy at edge point in high-frequency sub-bands. After decomposition of source modalities using 2-D RWT, it gives separated $+45^{\circ}$ and $-45^{\circ}$ orientations along with orientations provided by 2 -D DWT. The edge points in spectral domain are considered as reference points. The entropy of sub-block at edge point is estimated using Equation 9. Here, $w(i, j)$ is sub-block of size $m \times n$ centered at edge coefficient.

$$
\operatorname{En}(x, y)=\sum_{i=0}^{m-1} \sum_{j=0}^{n-1}|f(x+i, y+j)|^{2} w(i, j)
$$

The cross correlation (CCR) between entropy estimate at edge coefficient for both CT and MRI is computed using Equation 10. Here, ' $K$ ' stands for detailed sub-bands i.e. $A D, D A$, and $D D$.

$$
C C R^{C T, M R I}(x, y)=\frac{\sum_{i} \sum_{j} C T^{K}(x+i, y+j) M R I^{K}(x+i, y+j)}{E^{A}(x, y)+E^{B}(x, y)}
$$

The value of CCR represents significance of edge coefficient. If CCR is higher than a certain margin, the source coefficients in both the images are significant and needs to be considered in composite plane. In this case, fusion 
of coefficients are considered using Equation 11. Here, $\alpha \epsilon(0,1)$ is constant and its value is maximum if entropy of sub-block in CT is high and vice versa.

$$
\text { NewPlane } e^{K}(u, v)=\alpha C T^{K}(u, v)+(1-\alpha) M R I^{K}(u, v)
$$

If CCR value is smaller, the edge coefficient with high entropy is selected in composite plane as defined by Equation 12. Here, $E n_{C T}^{K}$ and $E n_{M R I}^{K}$ are the entropy estimations at edge point in $K^{\text {th }}$ detailed plane for CT and MRI, respectively. Here, ' $K$ ' stands for detailed sub-bands i.e. $A D, D A$, and $D D$.

$$
\operatorname{NewPlane}^{K}(u, v)=\left\{\begin{array}{l}
C T^{K}(u, v) \text { for } E n_{C T}^{K} \geq E n_{M R I}^{K} \\
M R I^{K}(u, v) \text { for } E n_{C T}^{K}<E n_{M R I}^{K}
\end{array}\right.
$$

\section{Results and Discussions}

The experiments are performed on axial brain images in two modalities namely CT and MRI. The study sets are selected from the same patient. The slices are marked for fusion with the help of expert radiologist considering the structural cues as a reference. Simens CT scan - Somatom Spirit scanner and Siemens 1.5 T MRI - Magnetom Cl machine are used to acquire few study sets of CT and MRI images, respectively. The CT scans and MRI slices are used with various sizes as 512 x 512, 512 × 304, 256 × 256, 1024 x 1024, etc. Other sample sets used in the experiments are from two websites viz. 'https://metapix.de/toolbox.html' and 'https://radiopaedia.org/'. Figure 5 displays few sample sets used to evaluate the performance of the proposed algorithm. All the computations are done in MATLAB2013a on the personal computer with specifications as Processor - Intel (i5$3210 \mathrm{MB}$ ), CPU speed - $2.50 \mathrm{GHz}$, and RAM - $4 \mathrm{~GB}$. The fused images are evaluated for its visual quality using objective and subjective evaluations.

\subsection{Objective Evaluation}

The performance of proposed algorithm is evaluated using objective fusion metrics namely Entropy (En), Root Means Square Error (RMSE), Fusion Factor (FusFac), Edge Quality Measure $\left(E Q_{A, B}^{f}\right.$ ), and mean Structural Similarity Index Measure ( $m S S I M)$. The quality of fused image is considered to be better, if En, FusFac have higher values, RMSE is smaller in value, and the parameters $E Q_{A, B}^{f}, m S S I M$ must approach to 'one'. These objective evaluation parameters are presented as follows:

Entropy (En) is a fusion metric which represents the content of fused image effectively. It is the energy representation of an image computed using Equation 13. Here, pi stands for gray level distribution in an image. The fused image is considered as a better image, if it gives higher value of entropy.

$$
E n=\sum_{i=0}^{N-1} p_{i} \log _{2} \frac{1}{p_{i}}
$$

Root Means Square Error (RMSE) is measure to evaluate quality of fused image in comparison with source images. The average value of RMSE of fused image with CT and MRI is taken as an estimate. Lower value of RMSE means the fused image carry all relevant details from source images. The RMSE is estimated using Equation 14. Here, $f_{s}(x, y)$ is source image and $f_{f u s}(x, y)$ is fused image.

$$
\operatorname{RMSE}(x, y)=\left[\frac{1}{M N} \sum_{x=0}^{M-1} \sum_{y=0}^{N-1}\left|f_{s}(x, y)-f_{f u s}(x, y)\right|^{2}\right]^{\frac{1}{2}}
$$

Fusion Factor (FusFac) is a measure of mutual information between source images and fused image. It is given by Equation 15. Here, $M I_{A, B}$ represents mutual information of two source image A and $\mathrm{B}$, whereas $M I_{\text {fus }}$ is mutual information of the fused image. The fused image is declared as better quality image, if value of fusion factor $(F u s F a c)$ is higher.

$$
\text { FusFac }=M I_{A, B}+M I_{\text {fus }}
$$

Edge Quality Measure $\left(E Q_{A, B}^{f}\right)$ is measure of preserving edges in fused image. It is estimated using Equation 16. Here, $f$ is fused image and A, B are source image slices. The 'zero' value of $E Q_{A, B}^{f}$ indicates loss of edges in the fused image and 'one' indicates a high rate of preserving edges from source images [21]. 

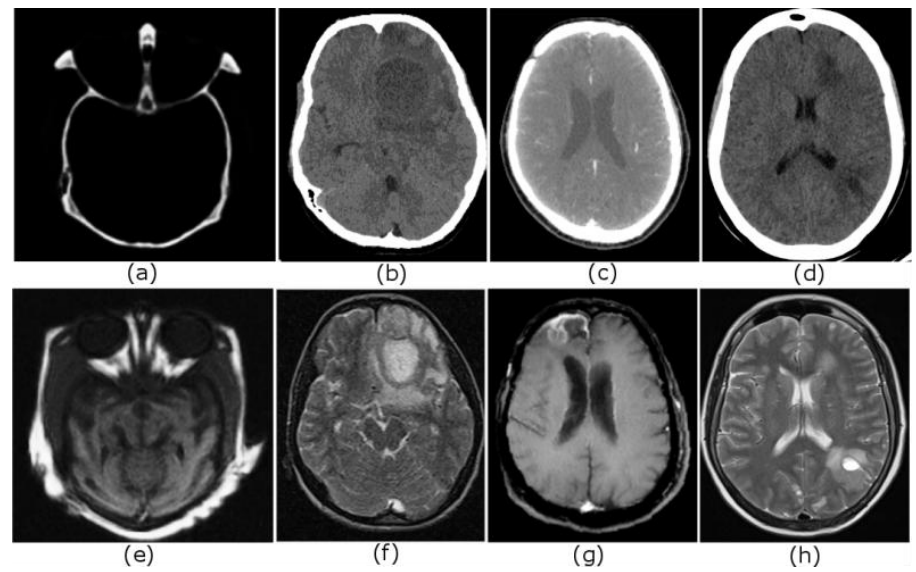

Fig. 5. Study sets of brain slices used in experiments - CT scans: (a) Set 1 (b) Set 2 (c) Set 3 (d) Set 4, and MRI slices: (e) Set 1 (f) Set 2 (g) Set 3 (h) Set 4

$$
E Q_{A, B}^{f}=\frac{\sum_{x=0}^{M-1} \sum_{y=0}^{N-1} E Q_{A, f}(x, y) w_{a}(x, y)+E Q_{B, f}(x, y) w_{b}(x, y)}{\sum_{x=0}^{M-1} \sum_{y=0}^{N-1} w_{a}(x, y)+w_{b}(x, y)}
$$

Mean Structural Similarity Index Measure (mSSIM) provides structural similarities between source images and fused image. It is estimated using Equations 17 and 18. Here, $\mu$ is the mean and $\sigma$ is the standard deviation. The iterative parameters used for computation are $\mathrm{C}_{1}=6.50$ and $\mathrm{C}_{2}=58.52$. The value 'one' for $m$ SSIM indicates that the fused image carry almost all relevant structural content from source images [1], [19].

$$
\begin{aligned}
& \operatorname{SSIM}(i, j)=\frac{\left(2 \mu_{i} \mu_{j}+C_{1}\right)\left(2 \sigma_{i j}+C_{2}\right)}{\left(\mu_{i}^{2}+\mu_{j}{ }^{2}+C_{1}\right)\left(\left(\sigma_{i}{ }^{2}+\sigma_{j}^{2}\right)+C_{2}\right.} \\
& \operatorname{mSSIM}(i, j)=\frac{1}{M} \sum_{k=1}^{M} \operatorname{SSIM}\left(i_{k}, j_{k}\right)
\end{aligned}
$$

The proposed algorithms is compared with other fusion algorithms such as pixel average method, Principal Component Analysis (PCA), Discrete Wavelet Transform (DWT), and Rotated Wavelet Transform (RWT) with maxima fusion rule. Table 1 shows the comparative analysis of these algorithms using objective evaluation parameters for four sample study sets. The value of entropy $(E n)$ and fusion factor $(F u s F a c)$ are the highest for proposed algorithm in all the sample sets. The value of parameter RMSE is the smallest one and the values of $\left(E Q_{A, B}^{f}\right)$ and mSSIM are higher for the proposed RWT algorithm compared to other algorithms. Thus objective evaluation reveals that the fused image using proposed algorithm is superior in terms of visual quality over other fusion methods.

\subsection{Subjective Evaluation}

The fused images are also evaluated subjectively with the help of expert radiologists. The opinion of radiologists is very important as they are end user of the proposed algorithm for cancer patient management. The fused images are shown to the radiologists and asked them to rate the quality of the image in comparison with source images.

The subjective score provided for the rating the quality of an image is ' 0 ' for poor content and ' 5 ' for excellent content in the fused image. The score is calculated as an average score for 10 different study sets evaluated by three radiologists. The average score of subjective analysis of the fused image with various fusion algorithms is presented in Table 2.

It is found that the average score is 3.80 i.e. the highest for fused images using proposed algorithm. It signifies that the fused images using proposed algorithm are better quality images and useful in cancer treatment. The fused images shows almost all anatomical structures without visual artifacts from both the modalities.

Figure 6 shows the result images for Set 1 (first row) and Set 2 (second row). Fused images with RWT maxima and proposed algorithm are visually better compared to other algorithms. They carry both bony structures and soft tissue representations prominently. Figure 7 displays fused images for the study set 3 (first row) and 4 (second row).

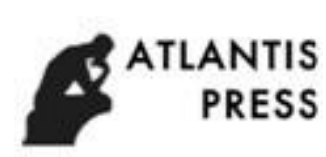


It is observed that the fused images using RWT algorithm are better quality images as compared with other spatial domain and wavelet domain techniques. Fused images with proposed technique are high contrast images with almost no visual artifacts. Thus these result images are useful in better visualization of lesions keeping anatomical structures undistorted. The fused images with proposed algorithm shows better textural content due to 'maxima' fusion rule in the approximate spectral plane. It also preserves the edge related content due to blockwise entropy fusion rule at edge points in detailed spectral planes. The radiologists also confirm the better visual quality of fused images using the proposed algorithm.

The fused images are also useful to the radiologists in delineation of lesions during treatment planning and to review the effectiveness of treatment in follow up examinations.

Table 1. Comparative objective analysis of the proposed algorithm with other fusion techniques

\begin{tabular}{llccccc}
\hline Study Set & Algorithm & $E n$ & RMSE & FusFac & $E Q$ & $m$ SSIM \\
\hline \multirow{5}{*}{ Set 1 } & Pixel Average & 5.7317 & 8.5271 & 4.9441 & 0.4128 & 0.7365 \\
& PCA & 6.6140 & 9.7726 & 6.0502 & 0.4311 & 0.7697 \\
& DWT maxima & 5.9952 & 8.9546 & 5.1460 & 0.5610 & 0.8218 \\
& RWT maxima & 7.0507 & 8.1253 & 5.5936 & 0.6158 & 0.8345 \\
& Proposed & 7.9548 & 7.9277 & 6.1248 & 0.7029 & 0.9271 \\
\hline \multirow{5}{*}{ Set 2 } & Pixel Average & 5.2269 & 5.5831 & 4.4973 & 0.5981 & 0.4922 \\
& PCA & 6.4032 & 7.0762 & 4.9774 & 0.6152 & 0.5496 \\
& DWT maxima & 6.4968 & 4.7542 & 5.0500 & 0.6871 & 0.6387 \\
& RWT maxima & 6.8247 & 4.0070 & 5.8674 & 0.6987 & 0.6377 \\
& Proposed & 7.4512 & 3.3258 & 6.3521 & 0.8987 & 0.7377 \\
\hline \multirow{5}{*}{ Set 3 } & Pixel Average & 4.9238 & 4.1631 & 3.9506 & 0.7194 & 0.3802 \\
& PCA & 6.3867 & 7.5921 & 4.3768 & 0.7258 & 0.6040 \\
& DWT maxima & 6.6732 & 6.5698 & 4.3331 & 0.8605 & 0.5449 \\
& RWT maxima & 6.4977 & 5.8422 & 3.6949 & 0.8694 & 0.5598 \\
& Proposed & 7.1463 & 4.1257 & 5.0347 & 0.9194 & 0.6107 \\
\hline \multirow{5}{*}{ Set 4 } & Pixel Average & 6.3867 & 7.5921 & 4.3768 & 0.6307 & 0.6039 \\
& PCA & 4.9238 & 6.1631 & 3.9506 & 0.6453 & 0.3802 \\
& DWT maxima & 6.4741 & 6.1853 & 5.7354 & 0.6529 & 0.5834 \\
& RWT maxima & 6.4977 & 6.8422 & 3.6949 & 0.7337 & 0.5598 \\
& Proposed & 7.1056 & 5.1258 & 6.6949 & 0.7941 & 0.6197 \\
\hline \multirow{5}{*}{} & & & & & &
\end{tabular}

Table 2. Average score by radiologists on visual quality of fused images using various fusion algorithms.

\begin{tabular}{|c|l|c|c|c|c|}
\hline \multirow{2}{*}{$\begin{array}{c}\text { Sr. } \\
\text { No. }\end{array}$} & \multirow{2}{*}{ Algorithm } & \multicolumn{4}{|c|}{ Subjective Score by radiologists } \\
\cline { 3 - 6 } & & $\# 1$ & $\# 2$ & $\# 3$ & Average \\
\hline 1 & Pixel Average & 2.50 & 2.80 & 2.70 & 2.67 \\
\hline 2 & PCA & 2.70 & 3.00 & 3.20 & 2.97 \\
\hline 3 & DWT maxima & 2.90 & 3.10 & 3.30 & 3.10 \\
\hline 4 & RWT maxima & 3.10 & 3.30 & 3.40 & 3.27 \\
\hline 5 & Proposed & 3.60 & 3.80 & 4.00 & 3.80 \\
\hline
\end{tabular}




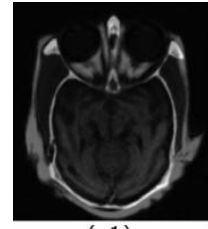

(a1)

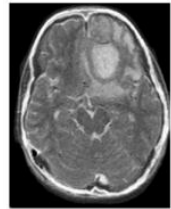

(a2)

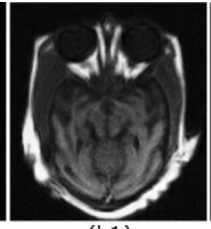

(b1)

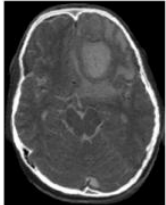

(b2)

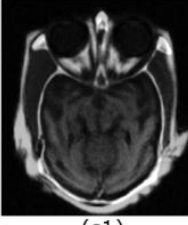

(c1)

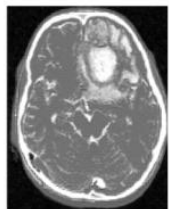

(c2)
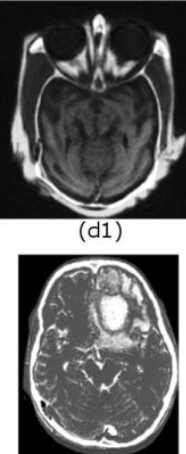

(d2)

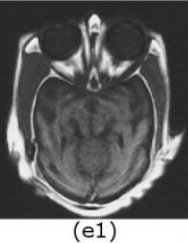

(e1)

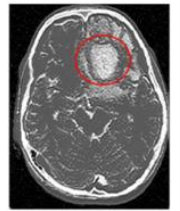

(e2)

Fig. 6. Fused images for study set 1 (first row) and 2 (second row) for algorithms: (a1, a2) Pixel Average (b1, b2) PCA (c1, c2) DWT maxima (d1, d2) RWT maxima (e1, e2) Proposed.
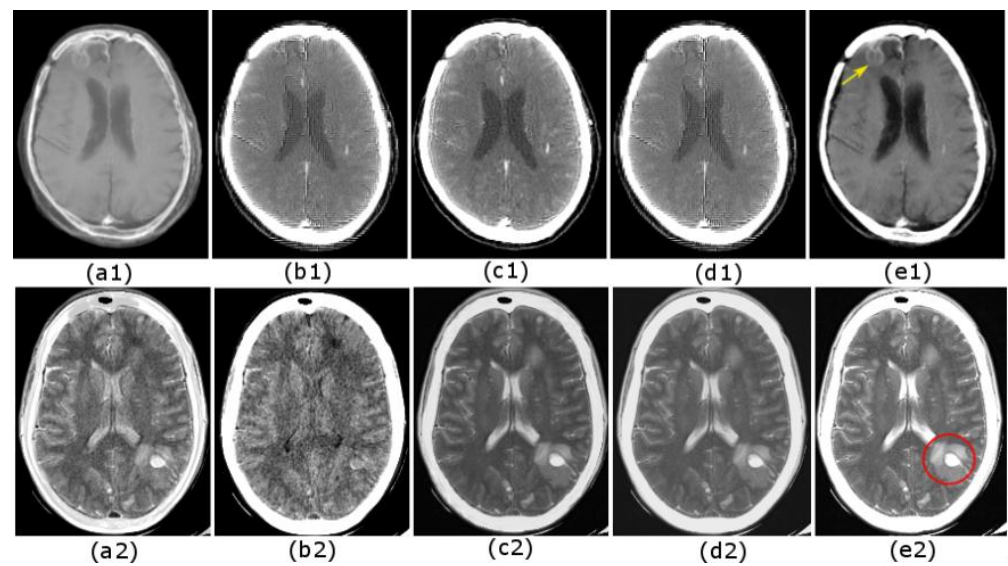

Fig. 7. Fused images for study set 3 (first row) and 4 (second row) for algorithms: (a1, a2) Pixel Average (b1, b2) PCA (c1, c2) DWT maxima (d1, d2) RWT maxima (e1, e2) Proposed.

\section{Conclusions}

This paper presents a new feature based fusion algorithm for CT and MRI images using Rotated Wavelet Transform. The edge related features are efficiently combined using maxima and entropy based fusion rules. The fused images using proposed RWT algorithm are visually better in terms of preserving texture and edge-related information from source modalities and display high contrast. The average subjective evaluation score is 3.80 given by expert radiologists suggests that the fused images using proposed algorithm are much useful in cancer treatment through radiotherapy. The objective evaluation using fusion parameters also has strong correlation with subjective opinion of radiologists. The proposed algorithm outperforms in fusion metrics over other fusion algorithms.

The proposed work may be validated on large study sets which will help in evaluating its robustness in cancer patient management system. The orientations presented by RWT are limited and algorithms which will explore more directionality can be developed in future.

\section{Acknowledgement}

Authors are very thankful to the Department of Radio diagnosis in S. K. N. Medical College and General Hospital, Narhe, Pune, Maharashtra, India for extending their help with test images for validating the proposed algorithm and providing the subjective evaluation of the fused images. Authors are also thankful to websites 'https://metapix.de/toolbox.html' and 'https://radiopaedia.org/' from where few sample sets are taken to evaluate the performance of the proposed algorithm.

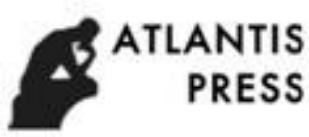




\section{References}

[1] Biswas, R., Sarawadekar, K., Varma, S., Banerjee, S.: An FPGA-based architecture of DSC-SRI units specially for motion blind ultrasound systems. Journal of Real Time Image Processing, 10, 573-595 (2015)

[2] Chavan, S. S., Talbar, S. N.: Multimodality image fusion in the frequency domain for radiation therapy. In: IEEE International Conference on Medical Imaging, m-Health and Emerging Communication Systems (MedCom), pp. 174-178, IEEE Press, New York (2014)

[3] Das, S., Kundu, M. K.: NSCT based multimodal medical image fusion using pulse coupled neural network and modified spatial frequency. Medical and Biological Engineering and Computing, 50, 11051114 (2012)

[4] Das, S., Kundu, M. K.: A neuro-fuzzy approach for medical image fusion. IEEE Transactions on Biomedical Engineering, 60, 3347-3353 (2013)

[5] Daubechies, I.: Ten Lectures on Wavelets. Society for Industrial and Applied Mathematics, (1992)

[6] James, A.P., Dasarathy, B. V.: Medical image fusion: A survey of the state of the art. Elsevier Information Fusion, 19, 4-19 (2014)

[7] Jorg, P.: Advanced Imaging in Biology and Medicine: Technology, Software Environments, Applications. Springer, Heidelberg (2009)

[8] Kavitha, C.T., Chellamuthu, C.: Multimodal medical image fusion based on Integer Wavelet Transform and neuro-fuzzy. In: IEEE International Conference on Signal and Image Processing (ICSIP), pp. 296300, IEEE Press, New York (2010)

[9] Kokare, M., Biswas, P. K., Chatterji, B. N.: Texture image retrieval using rotated wavelet filters. Pattern Recognition Letters, 28, 1240-1249 (2007)

[10] Mallat, S. G.: A theory for multiresolution signal decomposition: the wavelet representation. IEEE Transactions on Pattern Analysis and Machine Intelligence, 11, 674-693 (1989)

[11] Nikolov, S., Hill, P., Bull, D., Canagarajah, N.: Wavelets in Signal and Image Analysis: From Theory to Practice. Springer, Heidelberg (2001)

[12] Pradhan, P.S., King, R.L., Younan, N.H., Holcomb, D.W.: Estimation of the number of decomposition levels for a wavelet-based multiresolution multisensor image fusion. IEEE Transactions on Geoscience and Remote Sensing, 44, 3674-3686 (2006)

[13] Rajkumar, S., Kavitha, S.: Redundancy discrete wavelet transform and contourlet transform for multimodality medical image fusion with quantitative analysis. In: Third International Conference on Emerging Trends in Engineering and Technology, pp. 134-139, IEEE Computer Society (2010)

[14] Selesnick, I. W., Baraniuk, R. G., Kingsbury, N. G.: The dual-tree complex wavelet transform (DTCWT). IEEE Signal Processing Magzine, 22, 123-151 (2005)

[15] Singh R., Khare, A.: A wavelet based multimodal medical image fusion. In: International Symposium on Medical Imaging: Perspectives on Perception and Diagnostics, in conjunction with Seventh Indian Conference on Computer Vision, Graphics and Image Processing (ICVGIP-2010) (2010)

[16] Singh, R., Srivastava, R., Prakash, O., Khare, A.: Multimodal medical image fusion in dual-tree complex wavelet transform domain using maximum and average fusion rules. Journal of Medical Imaging and Health Informatics, 2, 168-173 (2012)

[17] Wang, G., Feng Liu, Fenglin Liu, Cao, G., Gao, H., Vannier, M.W.: Design proposed for a combined MRI/computed-tomography scanner. Biomedical Optics and Medical Imaging (2013) doi:10.1117/2.1201305.004860.

[18] Wang, X.S., Li, C.H.G., Qiu, Z.X.J., Feng, Y.: A comparative study of three CT and MRI registration algorithms in nasopharyngeal carcinoma. Journal of Applied Clinical Medical Physics, 10, 3-10, Spring, Heidelberg (2009)

[19] Wang, Z., Bovik, A.C., Sheikh, H.R., Simoncelli, E.P.:Image quality assessment: from error visibility to structural similarity. IEEE Transactions on Image Processing, 13, 600-612 (2004)

[20] Webster, G.J., Kilgallon, J.E., Ho, K.F., Rowbottom, C.J., Slevin, N.J., Mackay, R.I.: A novel imaging technique for fusion of high-quality immobilised MR images of the head and neck with CT scans for radiotherapy target delineation. The British Journal of Radiology, 82, 497-503, (2009)

[21] Xydeas, C. S., Petrovic, V.: Objective image fusion performance measure. Electronics Letters, 36, 308309 (2000)

[22] Yang, Y., Huang, S.Y., Gao F.J.: Multi-focus image fusion using an effective discrete wavelet transform based algorithm. Measurement Science Review, 14, 102-108 (2014)

[23] Yang, Y., Park, D. S., Huang, S., Rao, N.: Medical image fusion via an effective wavelet-based approach. EURASIP Journal on Advances in Signal Processing, 2010, 13 pages (2010)

[24] Zaidi, H., Mawlawi, O., Orton C. G.: Simultaneous PET/MR will replace PET/CT as the molecular multimodality imaging platform of choice. Medical Physics, 34, 1525-1528 (2007) 